\title{
Adaptive Vertical Handoff Management Architecture
}

\author{
Faraz Idris Khan and Eui Nam Huh ${ }^{*}$ \\ Internet Computing and Security Lab \\ Department of Computer Engineering, Kyung Hee University, 449-701 Suwon, South Korea \\ \{faraz, johnhuh\}@khu.ac.kr
}

\begin{abstract}
The 4G mobile system is a collection of radio networks providing access to IP based services. It ensures seamless roaming and users are always connected to the best network providing QoS (Quality of Service) to the end user. The mechanism of switching to a different network is termed as vertical handoff. In this paper we propose a vertical handoff management architecture which ensures efficient utilization of a mobile terminal resource i.e. CPU. This is achieved by employing feedback mechanism which allocates CPU resource according to the network load by adapting the CPU scheduler. In the end the simulation results are presented which are in accord with our idea.
\end{abstract}

Keywords: feedback scheduling, CPU scheduler adaptation, quality of service, resource management, vertical handoff.

\section{Introduction}

The interest in $4 \mathrm{G}$ networks is increasing at a rapid pace as wireless networks and mobile communications are growing at an astonishing rate. It promises to provide a broader range, lower access costs, the convenience of using a single "all in one" device. This is achieved by overlaying two or more networks with differing characteristics. One of the advantages of such architecture is that it enables applications to maintain QoS (Quality of Service) by switching to a better network. There are two kinds of vertical handoff situation that might occur, low data rate to high data rate or vice versa. The application consumption rate should be adaptively adjusted proportional to the arrival rate of the packets by changing the CPU allocation. In order to provide undisrupted service to the end user there are various vertical handoff management architecture proposed in literature [1] which mostly considers content adaptation after vertical handoff. There is relatively no architecture proposed which consider the impact of vertical handoff on CPU usage. Section 2 discusses briefly the system architecture with our proposed Adaptive CPU scheduling module which is discussed in section 2.1. Section 3 discusses the simulation results.

\section{System Architecture}

The architecture is designed with a middleware based approach. The device, application, user, current network profile are stored in a context repository (CR). The decision

\footnotetext{
* Corresponding author.
} 
engine (DE) monitors the context cache for triggering the handoff upon detection of context changes i.e. which can be degradation in QoS due to changes in network characteristics, disconnection from the current network etc. Network Resource Monitoring Agent (NRMA) monitors the network resources i.e. packet arrival rate. The interaction among the modules is shown in figure 1. The CPU scheduler adaptation module is discussed in detail in subsection 2.1.

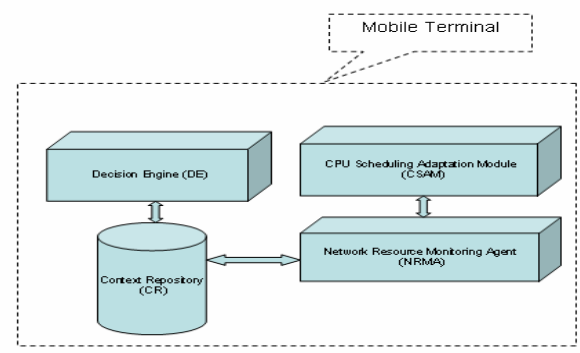

Fig. 1. System Architecture designed using a middleware based approach

\subsection{CPU Scheduler Adaptation Module}

A mobile terminal can either move from high data rate to low data rate or low data rate to high data rate. In case of first scenario the application consumes the data from the buffer at a faster rate than the arrival rate of the packets. This means that the application is allocated more CPU than needed. In the later case the buffer will fill up at a rate faster than the application consumption rate. The monitoring is done after an interval $\mathrm{t}$ which we call an epoch. An optimal point $q$ is defined in a buffer which is achieved by dynamically changing the CPU allocation $C P U_{\text {alloc }}$ proportional to the arrival rate of the packets.

We define two parameters buffer fill level and proportional change to implement CPU scheduler adaptation module.

Buffer fill level: The buffer fill level $\mathrm{q}(\mathrm{t})$ at time $\mathrm{t}$ is given by the formula

$$
\mathrm{q}(\mathrm{t})=\frac{\text { Current buffer size at time t }}{\text { Total buffer size }}
$$

Proportional change: Let us represent the arrival rate of the packets in the current epoch as $\lambda_{\text {current }}$ and the arrival rate of the packets in the previous epoch as $\lambda_{\text {previous }}$. Then proportional change $\mathrm{p}(\mathrm{t})$ at time $\mathrm{t}$ is given by the formula

$$
\mathrm{p}(\mathrm{t})=\frac{\lambda_{\text {current }}}{\lambda_{\text {previous }}}
$$

The algorithm for CPU scheduler adaptation module is given in Figure 2. 


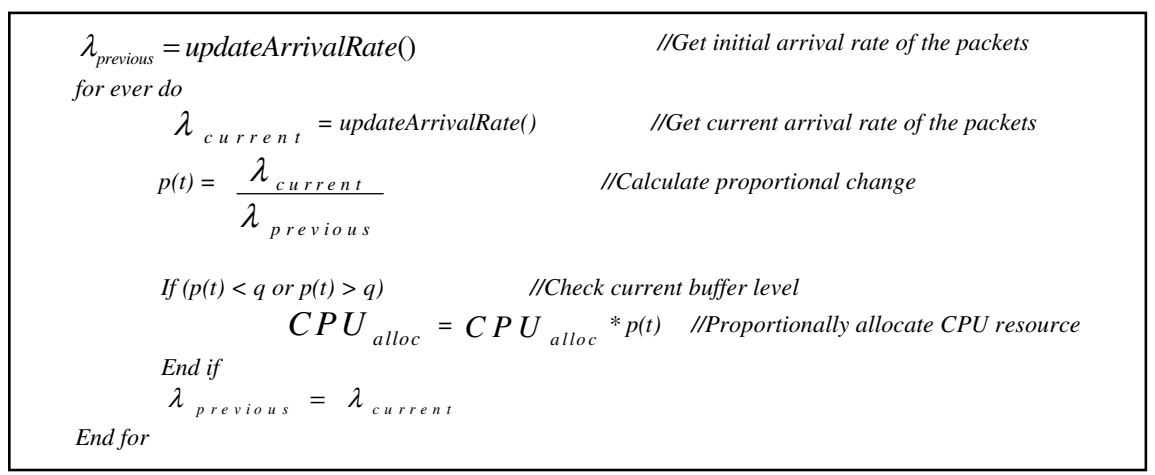

Fig. 2. CPU Scheduling Adaptation Logic

\section{Simulation}

The simulation considers the following vertical handoff scenarios

- Switching from low data rate network to high data rate network

- Switching from high data rate network to low data rate network

We simulated our idea in Discrete Event Simulation using C++. For our simulation we have considered the optimal buffer size of 100 packets. Table 1 shows the parameters that we have chosen to simulate the vertical handoff situation.

\subsection{Low Data Rate Network to High Data Rate Network}

When a mobile terminal switches to a network of high data rate the queue length will increase with the passage of time due to increase in arrival rate of the packets. From Figure 3 it is evident that the buffer size remains optimal during vertical handoff scenario and interestingly the average waiting time experienced by the packet also converges as shown in Figure 3.
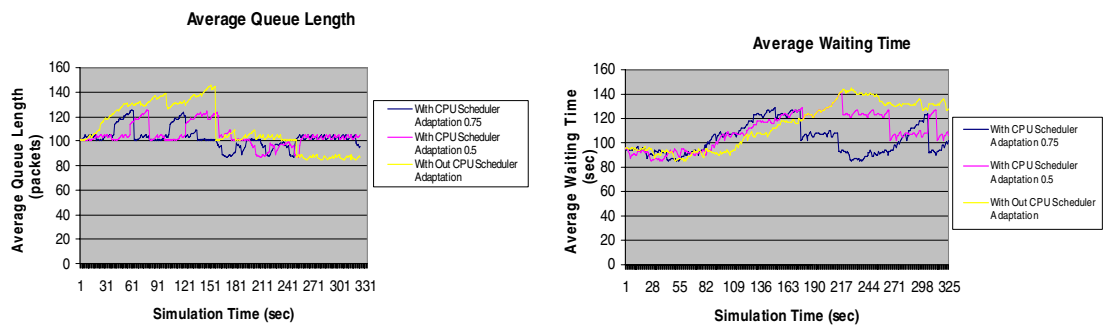

Fig. 3. Left side shows average queue length and right side shows average waiting time (Vertical handoff from low data rate to high data rate network) 


\subsection{High Data Rate Network to Low Data Rate Network}

In case of moving to a network with low data rate the queue size will decrease which is shown in figure 4 . The standard deviation from the optimal point with out CPU scheduling adaptation is 40 and with CPU scheduler adaptation the standard deviation is 15.3 and 18 for a proportional change of 0.75 and 0.50 respectively. The graph on the right hand side of Figure 4 shows the average waiting time of the packets in the queue in this scenerio.
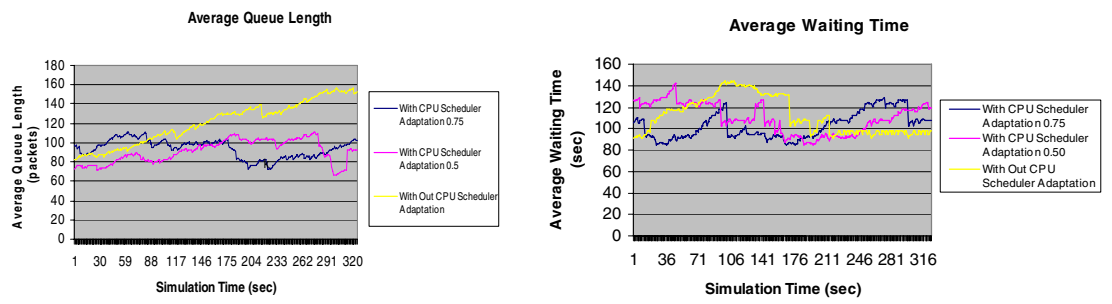

Fig. 4. Left side shows average queue length and right side shows average waiting time (Vertical handoff from high data rate to low data rate network)

Acknowledgements. This research was supported by MIC (Ministry of Information and Communication), Korea, under ITRC (Information Technology Research Center) support program supervised by the IITA (Institute of Information Technology Advancement). (IITA-2006-C1090-0603-0040).

\section{Reference}

1. Helal, S., Lee, C., Zhang, Y.G., Richard III, G.G.: An Architecture for Wireless LAN/WAN Integration. Wireless Communication and Networking Conference Vol. 3. IEEE, Chicago (2000) 1035-1041 